\title{
Does response interference contribute to face composite effects?
}

\author{
Jennifer J. Richler and Olivia S. Cheung \\ Vanderbilt University, Nashville, Tennessee \\ Alan C.-N. Wong \\ Chinese University of Hong Kong, Hong Kong \\ AND \\ ISABEL GAUTHIER \\ Vanderbilt University, Nashville, Tennessee
}

\begin{abstract}
Holistic processing of faces can be measured as a failure of selective attention to one face-half under instructions to ignore the other face-half in a naming or same/different matching task. But is interference from the irrelevant half due to response interference rather than to holistic processing? Here, participants learned to name two faces "Fred" and two "Bob." At test, composites were created from top and bottom halves of different learned faces or of a novel face, and composites were either aligned or misaligned. Naming was slower when the irrelevant half was from a different face as opposed to the same face, regardless of whether it was associated with the same name, a different name, or no name, suggesting holistic processing. Interference was eliminated when composite halves were misaligned. These results suggest that, unlike Stroop effects, composite effects are not due to response interference.
\end{abstract}

All faces share the same basic features (eyes, nose, mouth) in the same general, or "first-order," configuration. For this reason, the individuation of faces relies on the processing of "second-order" configural differences that include spatial relations between face parts (Diamond \& Carey, 1986; Maurer, Le Grand, \& Mondloch, 2002). It has been suggested that attention to small metric differences between distant face parts encourages the use of a holistic processing strategy. Indeed, a considerable amount of work suggests that faces are processed not in terms of individual features, but as wholes (Farah, Wilson, Drain, \& Tanaka, 1998; Le Grand, Mondloch, Maurer, \& Brent, 2004; Mondloch, Le Grand, \& Maurer, 2002; Tanaka \& Farah, 1993).

Holistic processing of faces is robust in a variety of conditions, such that participants are unable to selectively attend to a face part even when explicitly instructed to do so and when holistic processing is disadvantageous to performance. Such holistic effects are often demonstrated in a composite paradigm in which stimuli are made by combining the top half of one face with the bottom half of another face. In the original study using the composite task (Young, Hellawell, \& Hay, 1987), parts of famous faces were used to create stimuli. Participants had to name half of a composite face and ignore the identity of the irrelevant half. For example, the target part might have belonged to Brad Pitt but the irrelevant part might have belonged to Matt Damon. The inconsistency of the two parts led to slower RTs in identifying the relevant part.

Interference in this task is assumed to reflect holistic processing - the irrelevant part affects performance because the face is automatically processed as a whole. However, an alternative possibility is that the different responses associated with the target and irrelevant facehalves ("Brad Pitt" vs. "Matt Damon") compete, resulting in response interference analogous to classic Stroop effects, where, for example, the response to the word interferes with the response to the ink color (MacLeod, 1991). Response interference in Stroop paradigms is thought to arise when features that are processed in parallel lead to conflicting outputs (Cohen, Dunbar, \& McClelland, 1990) or activate competing motor programs (Singer, Lappin, $\&$ Moore, 1975). To what extent is the composite effect due to competing responses for the target and irrelevant face-halves, rather than to holistic processing, where interference arises because face parts are not processed independently?

Since the original demonstration of the composite effect with famous faces, variants of the composite task have used unfamiliar faces in simultaneous matching (Hole, 1994) and sequential matching paradigms (e.g., Cheung, Richler, Palmeri, \& Gauthier, 2008; Farah et al., 1998; Gauthier, Curran, Curby, \& Collins, 2003; Goffaux \& Rossion, 2006; Le Grand et al., 2004; Michel, Rossion,

J. J. Richler, jennifer.j.richler@vanderbilt.edu 
Han, Chung, \& Caldera, 2006; Richler, Tanaka, Brown, \& Gauthier, 2008). In these cases, participants judge whether one half of a test face is the same as or different than the study face, and are told to ignore the other face-half. Holistic processing is inferred from better performance on congruent trials (both parts same/both parts different) than on incongruent trials (one part same, one part different; congruency effect; e.g., Cheung et al., 2008; Gauthier et al., 2003; Richler, Gauthier, Wenger, \& Palmeri, 2008). Response interference could also contribute in such a task: Although the face-halves do not have names, the response for the irrelevant part may still be different from the response for the target part (e.g., if the response to the target part is "same" when the irrelevant part is different). Indeed, it has been argued that the congruency effect in the matching composite task measures Stroop-like response interference rather than true holistic processing (Robbins \& McKone, 2007). However, the extent to which interference in the composite task is analogous to Stroop interference has never been addressed, and this is the goal of the present study.

To this end, participants learned to associate names with four composite faces. The names were randomly assigned: Two faces were named "Bob" and two others were named "Fred." In this way, we could create composites at test whereby the irrelevant part could be from a different studied face, but the response associated with the irrelevant part could be the same as or different than the target part. For example, the top of Bob 1 could be paired with the bottom of Bob 2, in which case any interference would be due to holistic processing (because the irrelevant part belongs to a different identity), but not to response interference (because the target and irrelevant part would elicit the same response ["Bob"]). Alternatively, the top of Bob 1 could be paired with the bottom of Fred 1, in which case we could observe response interference because the target and irrelevant parts are associated with different responses that may compete ("Bob" vs. "Fred") in addition to interference related to holistic processing. In addition to the four learned faces, parts from a fifth composite face that was not learned were also used. Thus, there were four types of irrelevant parts that could be paired with the target part to create composites at test (see Table 1): same face with the same name as the target part (SFSN); differ- ent face with the same name (DFSN); different face with a different name (DFDN); and an unfamiliar face not used during training (UF).

Each of these conditions was compared with a baseline condition in which the target part had to be named when shown in isolation, so there was no irrelevant part that could interfere with (or facilitate) performance. Consistent with the idea that the composite task measures holistic processing, we expect to find some degree of interference in all conditions in which the irrelevant face-half is from a different face than the target part, regardless of whether the irrelevant part is from a face that was previously studied (DFSN and DFDN) or unfamiliar (UF) - performance should be impaired when the irrelevant half contains information that is inconsistent with the target part.

More interestingly, we compared performance between the three conditions in which we expect interference (DFSN/DFDN/UF), to determine if response interference contributes to the amount of interference observed. If there is more interference when the irrelevant part is associated with a different name than the target part, as opposed to when the irrelevant part is associated with the same name as the target part, or when it has no name (DFDN vs. DFSN/UF), this would suggest a contribution of response interference.

Young et al. (1987) inferred holistic processing by comparing naming of face parts between aligned and misaligned composites (alignment effect). However, it was impossible to assess whether there was any remaining interference when face-halves were misaligned, because there was no baseline (for further discussion of the limitations of the alignment effect in measuring holistic processing, see Cheung et al., 2008; Gauthier \& Bukach, 2007). In fact, a recent study that measured holistic processing in terms of a congruency effect for both aligned and misaligned composite trials individually found that although holistic processing was reduced when face-halves were misaligned, it was not always eliminated (Richler, Tanaka, et al., 2008).

Interference may be observed even after face parts have been misaligned because misalignment reduces holistic processing (e.g., Goffaux \& Rossion, 2006; Robbins \& McKone, 2007; Young et al., 1987) but does not affect response interference. For example, even if misalignment

Table 1

Four Types of Irrelevant Parts That Could Be Paired With the Target Part to Create Composites During Phase 3 and Predictions for Each Condition

\begin{tabular}{|c|c|c|}
\hline Condition & Description & Predictions \\
\hline Same face/same name & $\begin{array}{l}\text { Both the target and irrelevant part } \\
\text { were from the same studied face. }\end{array}$ & $\begin{array}{l}\text { Congruent trials, no interference } \\
\text { expected }\end{array}$ \\
\hline Different face/same name & $\begin{array}{l}\text { The irrelevant part was from a dif- } \\
\text { ferent face that shared the same } \\
\text { name with the target part. }\end{array}$ & $\begin{array}{l}\text { Interference due to holistic pro- } \\
\text { cessing with a familiar face part }\end{array}$ \\
\hline Different face/different name & $\begin{array}{l}\text { The irrelevant part was from a dif- } \\
\text { ferent face that was assigned a dif- } \\
\text { ferent name than the target part. }\end{array}$ & $\begin{array}{l}\text { Interference due to holistic pro- } \\
\text { cessing with a familiar face part } \\
\text { plus any response interference }\end{array}$ \\
\hline Unfamiliar face & $\begin{array}{l}\text { The irrelevant half was from the } \\
\text { unfamiliar face not named or stud- } \\
\text { ied during training. }\end{array}$ & $\begin{array}{l}\text { Interference due to holistic process- } \\
\text { ing with an unfamiliar face part }\end{array}$ \\
\hline
\end{tabular}


reduces holistic processing because of an unfamiliar configuration of parts, the mere presence of the irrelevant face-half, regardless of the arrangement of parts, may trigger its associated response. Indeed, in classic Stroop tasks, separating the color patch and color name in space reduces, but does not eliminate, response interference (Gatti \& Egeth, 1978; MacLeod, 1991). Thus, we also test whether holistic processing, response interference, or both, are attenuated by misalignment, by comparing performance on the misaligned conditions with baseline. For completeness, we also report analyses on the alignment effect (difference between aligned and misaligned trials).

\section{METHOD}

\section{Participants}

Participants were 29 members of the Vanderbilt University community (13 male; mean age: 24.16 years; normal/corrected-tonormal vision) who provided informed consent and were compensated with $\$ 12$.

\section{Stimuli}

Five face tops and five face bottoms were selected from the Max Planck Institute face database (Troje \& Bülthoff, 1996) and randomly combined into five composite faces. For each participant, two of these composites were assigned the name "Bob" and two of the composites were named "Fred." Parts from the fifth composite were not assigned a name and were only used during testing. Name assignment was counterbalanced across participants.

Faces were approximately $5 \times 6.5 \mathrm{~cm}$, and a white line $2 \mathrm{~mm}$ thick separated top and bottom halves. When only one face-half was presented, the region either above or below the white line was all gray. Faces were presented in the middle of a gray rectangle $(17.6 \times$ $16.8 \mathrm{~cm}$ ) positioned centrally on a white background.

Misaligned composites were created by moving the top half of the composite leftward and the bottom half rightward, so that the edge of one face-half fell in the center of the other face-half.

\section{Procedure}

The experiment was conducted on iMac computers with a $640 \times$ 480 pixel resolution. There were three phases in the experiment.

In Phase 1 (whole-face training), participants learned the names of four whole composite faces. First, all four faces and their assigned names were displayed on the screen for participants to study for as long as they liked. Once participants terminated the study phase, training trials began. On each training trial, a fixation cross was presented $(500 \mathrm{msec})$, followed by a face. Participants were instructed to press " 1 " if the face was assigned the name "Bob" and to press " 2 " if the face was assigned the name "Fred." All participants completed three blocks of 40 trials. After the third block, participants were informed of their response time (RT) and accuracy. If accuracy was $95 \%$ or higher, with RTs less than $1,000 \mathrm{msec}$, participants moved on to Phase 2. Otherwise, participants completed another block of 40 trials until this criterion was achieved, to a maximum of three additional blocks.

In Phase 2 (part-naming training), participants practiced naming face-halves presented in isolation. Training was identical to Phase 1 , with the exception that a single face-half was presented on each trial. Participants named top halves until criterion was reached, and repeated the training procedure with bottom halves. Phase 2 was included to ensure that names were strongly associated with each learned part. If anything, it should only serve to reduce holistic processing and enhance response interference because of practice with isolated parts.

Phase 3 was the test phase. First, the four faces were again presented on the screen with their assigned names. On the test trials, a fixation was shown $(500 \mathrm{msec})$, followed by a composite face. Composites were created by pairing a target part with one of four possible irrelevant parts with respect to the target part (see Table 1). Irrelevant part conditions were randomized during the experiment. Participants were instructed to indicate the name of the target face-half (top or bottom) as accurately as possible using the same response keys as during training, while ignoring the irrelevant face-half. Face composites were either aligned or misaligned. Alignment was blocked within participants because previous work has shown that aligned faces can induce holistic effects for subsequently presented stimuli in instances in which they would otherwise not be observed (Richler, Bukach, \& Gauthier, 2009). Order was counterbalanced so that 15 participants were tested on aligned composites first, and 14 participants were tested on misaligned composites first. Baseline trials showing only the target face-half were included among both the aligned and misaligned test blocks, and we calculated a separate baseline for the aligned and misaligned conditions.

Participants were told at the beginning of each block which face part (top or bottom) was the target, and on each trial four dots surrounding the target half also provided a reminder. There were five blocks of top-naming trials and five blocks of bottom-naming trials that were alternated within each of the alignment blocks. In total, there were 480 trials.

\section{RESULTS}

Performance for Phases 1 and 2 (learning phases) is reported in Table 2. Mean correct RTs and accuracy for Phase 3 are shown in Figure 1. Trials for which RTs were beyond three standard deviations of the mean were discarded $(0.6 \%$ of trials).

\section{Response Time}

A $2 \times 5$ repeated measures ANOVA with factors alignment (aligned/misaligned) and condition (baseline/SFSN/ DFSN/DFDN/UF) revealed a main effect of alignment, with longer RTs for aligned than for misaligned composites $[F(1,28)=12.01, p<.01]$. There was also a main effect of condition $[F(4,112)=9.83, p<.0001]$ that was modulated by an interaction with alignment $[F(4,112)=$ $4.26, p<.01]$. Scheffe's tests revealed that for aligned composites, performance for DFSN, DFDN, and UF was significantly slower than baseline $(p s<.01)$ and there was no significant difference between these conditions $(p s>.5)$. For misaligned composites, no condition was significantly slower than baseline ( $p \mathrm{~s}>.6)$.

\section{Accuracy}

A $2 \times 5$ repeated measures ANOVA with factors alignment (aligned/misaligned) and condition (baseline/SFSN/ DFSN/DFDN/UF) showed no significant main effects. There was a trend toward a significant alignment $\times$ condition interaction $[F(4,112)=2.39, p=.055]$. Scheffé's

\section{Table 2}

Average Number of Blocks to Reach Learning Criterion and Average Accuracy and RT in the Last Block of Phase 1 (Whole Training) and Phase 2 (Part Training)

\begin{tabular}{cccc}
\hline $\begin{array}{c}\text { Learning } \\
\text { Condition }\end{array}$ & $\begin{array}{c}\text { No. Blocks } \\
\text { to Criterion }\end{array}$ & $\begin{array}{c}\text { Accuracy } \\
\text { (\% Correct) }\end{array}$ & RT (msec) \\
\hline Whole & 3.41 & 97.72 & 688.03 \\
Part & 3.45 & 96.83 & 637.79 \\
\hline
\end{tabular}



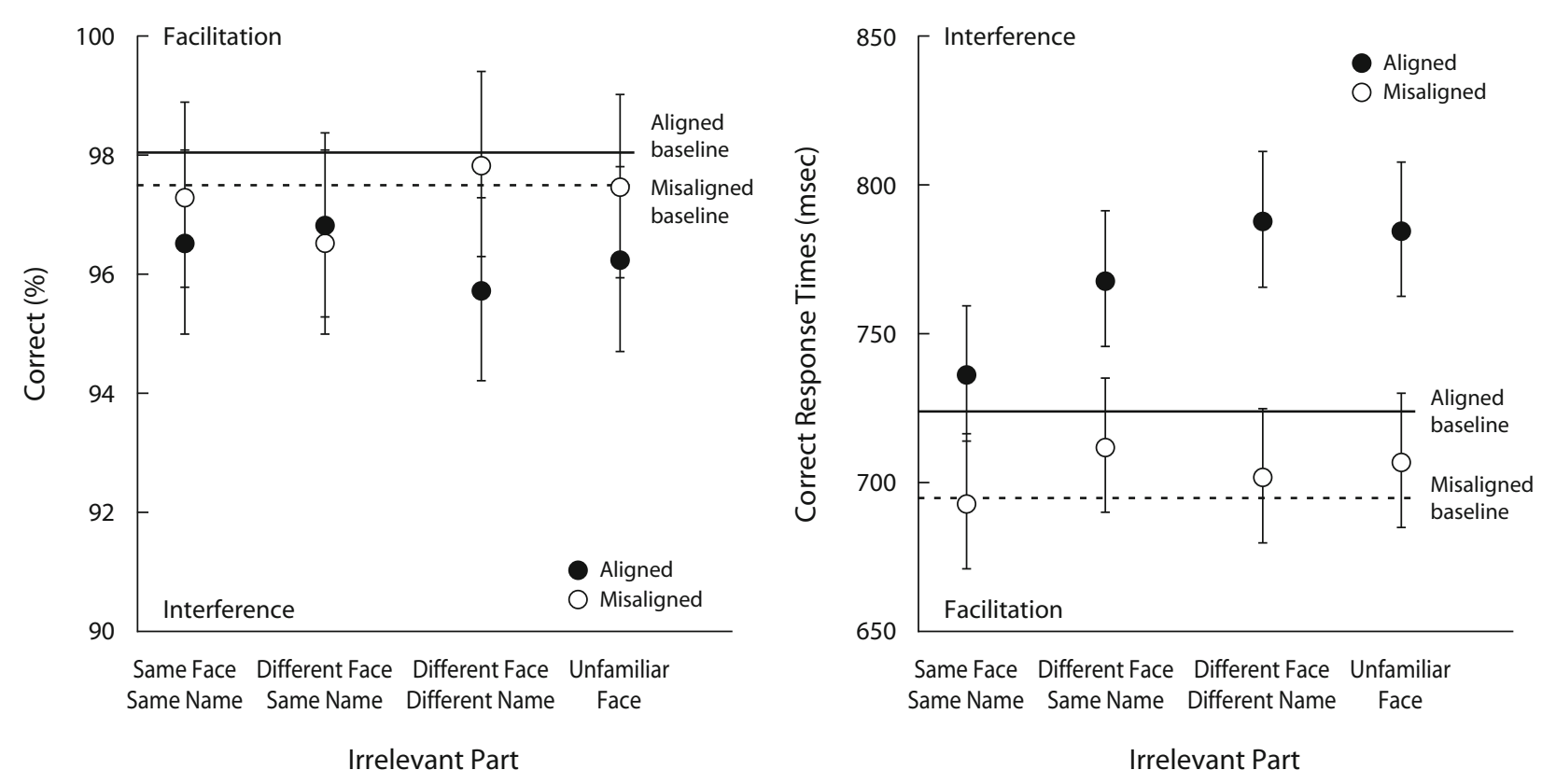

Figure 1. Accuracy (percent correct; left panel) and response times (in milliseconds; right panel) for naming the target half of aligned and misaligned composites as a function of the type of irrelevant part (SFSN/DFSN/DFDN/UF). Interference is determined by a significant difference in performance in comparison with the isolated baseline trials (solid line, aligned baseline; dotted line, misaligned baseline). Error bars show $95 \%$ confidence intervals of within-participants effects.

tests revealed that for aligned composites, only performance for DFDN was significantly worse than baseline $(p<.05)$. However, there was no significant difference between DFDN, DFSN, and UF ( $p$ s $>.6$ ). When composites were misaligned, no condition was significantly worse than baseline $(p s>.7)$.

\section{Alignment Effect \\ For each condition (baseline/SFSN/DFSN/DFDN/UF), we calculated an aligned-misaligned difference score and conducted a one-way repeated measures ANOVA on those values. In RT, there was a main effect of condition $[F(4,112)=4.26, p<.01]$. Scheffé's tests revealed that the alignment effect for DFDN was significantly larger than baseline $(p<.05)$, and the difference between UF and baseline approached significance $(p=.06)$. Impor- tantly, there was no significant difference between the alignment effect for the DFSN, DFDN, and UF conditions $(p s>.4)$. Analysis of the alignment effect in accuracy revealed no significant effects.}

\section{DISCUSSION}

For aligned composites, interference, as revealed by longer RTs relative to baseline, was observed in all conditions in which a different face-half (either novel or previously studied) was paired with the target half. In accuracy, performance was only significantly worse than baseline for the DFDN condition, suggestive of response interference-interference was only observed when the irrelevant part was associated with a different response than the target part. Moreover, the alignment effect was only significantly larger than baseline for the DFDN condition. However, we hesitate to interpret this as evidence of response interference for the following reasons. First, performance was significantly worse than baseline for all three interference conditions (DFSN/DFDN/UF) in RT, and the differences between DFDN and either DFSN or UF were not significant in RT, accuracy, or the alignment effect. Second, the accuracy data must be interpreted with caution due to potential ceiling effects. Finally, all interference effects (in RT and accuracy) were attenuated when composite face-halves were misaligned. In sum, our results suggest that the congruency effect measured in the composite task reflects holistic processing, with very little if any contribution of response interference.

According to a model by Cohen et al. (1990), the Stroop effect arises when stimulus features that are processed in parallel share a common mechanism. In the classic case of color naming and reading, the first shared resource is the response mechanism, so color-word Stroop effects arise due to response interference. However, interference in this model can arise at any stage of processing at which the target and irrelevant features rely on common mechanisms. Thus, in the composite task, Stroop-like interference that is not response interference could occur, since, in contrast to reading and color naming, face tops and face bottoms likely share processing resources prior to the response stage. However, according to the Cohen et al. model, interference arises because of more practice with one task (e.g., reading) than with the other (e.g., color naming), resulting in one processing pathway that is stronger and faster than the other. As such, the feature with which participants have more practice interferes with the 
less experienced feature, and not the other way around (Cohen et al., 1990; MacLeod \& Dunbar, 1988). Indeed, in bilingual participants, the Stroop effect is larger in their most proficient language because of a stronger association between the stimulus and response (Mägiste, 1984). Thus, from a Stroop perspective, an untrained irrelevant face-half should not lead to interference for a trained target face-half, since in this case participants have practice with the target half but not with the irrelevant half. However, our data do not show less interference for untrained vs. trained irrelevant halves, suggesting that interference in the composite task does not arise from competition between outputs associated with target and irrelevant face parts.

In contrast to their experience with Stroop stimuli, whereby participants have mostly independent experience with the target and irrelevant stimulus features (e.g., most of the time we read the words red or blue printed in black), participants in the composite task typically experience covariation between face parts. Outside of the lab, face parts change together. As Brad Pitt's mouth is replaced on the screen by Matt Damon's, the rest of the face can be assumed to change in the same way. Interference may arise with composite faces because they violate learned expectations that face parts change together. The idea that composite effects arise due to experience is supported by work with car experts (Gauthier et al., 2003) and with laboratory-trained experts with novel objects (Gauthier \& Tarr, 2002; Wong, Palmeri, \& Gauthier, in press), showing that both the congruency effect and its sensitivity to alignment increase with expertise.

Importantly, face parts may be processed together at either a perceptual level or a decisional level (Richler, Gauthier, et al., 2008). For example, holistic processing may arise due to the encoding of holistic representations in which face parts are not represented independently (Tanaka \& Farah, 1993). Alternatively, holistic processing might arise because, even though face parts are encoded and stored independently, they are not treated independently when decisions are made about them (Richler, Gauthier, et al., 2008; Wenger \& Ingvalson, 2002). Although our data suggest that interference indicative of holistic processing arises because face parts are processed together (rather than due to response or Stroop-like interference), they do not distinguish between perceptual and decisional holism. To address this question would require an analytical framework that defines perceptual and decisional loci for interactions between dimensions, such as the general recognition theory (Ashby \& Townsend, 1986). This is beyond the scope of this work. However, recent evidence suggests that there is an important decisional contribution to holistic processing of faces (see Richler, Gauthier, et al., 2008; Wenger \& Ingvalson, 2002).

Critically, response interference due to competition between response labels or motor programs is distinct from holistic processing, be it perceptual or decisional. Even if, as the decisional account of holism holds, face-halves are represented independently but the decisions made about the parts are dependent, this does not necessarily imply responses for each face-half that compete with each other. For example, holism might arise as decisional processes acting on the representations of each face part interact prior to response selection or the initiation of motor programs. Thus, finding no influence of response interference in the composite task should not be taken as evidence against the role of decisional factors in holistic processing of faces.

\section{CONCLUSIONS}

The composite face paradigm is similar in many ways to classic Stroop tasks: Performance is affected by irrelevant information in the stimulus, despite instructions to selectively attend to another dimension. In face composite experiments, we treat the top and bottom halves of faces as independent dimensions, and often construct stimuli in such a way that they are completely uncorrelated. Yet, the visual system does not appear to treat face parts independently, either because they are not independently represented or because of experience in making judgments about one face part that take another face part into consideration.

Our results demonstrate that interference measured in the composite task does not depend on experience with the irrelevant part and rejects an account of the composite effect whereby the response from the target half competes with the response from the irrelevant part, analogous to the Stroop effect. Instead, interference appears to arise because target and irrelevant face parts are not processed independently prior to response selection and execution.

\section{AUTHOR NOTE}

This research was supported by the James S. McDonnell Foundation, the National Eye Institute, the National Science Foundation (to I.G.), and the Temporal Dynamics of Learning Center (NSF Science of Learning Center SBE-0542013). The authors thank Stewart Phillips for running participants. Correspondence concerning this article should be addressed to J. J. Richler or I. Gauthier, Department of Psychology, Vanderbilt University, Wilson Hall, Nashville, TN 37203 (e-mail: jennifer.j.richler@ vanderbilt.edu or isabel.gauthier@vanderbilt.edu).

\section{REFERENCES}

Ashby, F. G., \& Townsend, J. T. (1986). Varieties of perceptual independence. Psychological Review, 93, 154-179.

Cheung, O. S., Richler, J. J., Palmeri, T. J., \& Gauthier, I. (2008). Revisiting the role of spatial frequencies in the holistic processing of faces. Journal of Experimental Psychology: Human Perception \& Performance, 34, 1327-1336.

Cohen, J. D., Dunbar, K., \& McClelland, J. L. (1990). On the control of automatic processes: A parallel distributed processing account of the Stroop effect. Psychological Review, 97, 332-361.

DiAmond, R., \& CAREY, S. (1986). Why faces are and are not special: An effect of expertise. Journal of Experimental Psychology: General, 115, 107-117.

Farah, M. J., Wilson, K. D., Drain, M., \& Tanaka, J. W. (1998) What is "special" about face perception? Psychological Review, 105, 482-498.

Gatti, S. V., \& Egeth, H. E. (1978). Failure of spatial selectivity in vision. Bulletin of the Psychonomic Society, 11, 181-184.

Gauthier, I., \& Bukach, C. (2007). Should we reject the expertise hypothesis? Cognition, 103, 322-330.

Gauthier, I., Curran, T., Curby, K. M., \& Collins, D. (2003). Per- 
ceptual interference supports a non-modular account of face processing. Nature Neuroscience, 6, 428-432.

GAUTHIER, I., \& TARR, M. J. (2002). Unraveling mechanisms for expert object recognition: Bridging brain activity and behavior. Journal of Experimental Psychology: Human Perception \& Performance, 28, 431-446.

Goffaux, V., \& Rossion, B. (2006). Faces are "spatial"-Holistic face perception is supported by low spatial frequencies. Journal of Experimental Psychology: Human Perception \& Performance, 32, 1023-1039.

HoLe, G. J. (1994). Configurational factors in the perception of unfamiliar faces. Perception, 23, 65-74.

Le Grand, R., Mondloch, C. J., Maurer, D., \& Brent, H. P. (2004). Impairment in holistic face processing following early visual deprivation. Psychological Science, 15, 762-768.

MacLeod, C. M. (1991). Half a century of research on the Stroop effect: An integrative review. Psychological Bulletin, 109, 163-203.

MacLeod, C. M., \& Dunbar, K. (1988). Training and Stroop-like interference: Evidence for a continuum of automaticity. Journal of Experimental Psychology: Learning, Memory, \& Cognition, 14, 126-135.

MäGISTE, E. (1984). Stroop tasks and dichotic translation: The development of interference patterns in bilinguals. Journal of Experimental Psychology: Learning, Memory, \& Cognition, 10, 304-315.

Maurer, D., Le Grand, R., \& Mondloch, C. J. (2002). The many faces of configural processing. Trends in Cognitive Sciences, 6, 255260.

Michel, C., Rossion, B., Han, J., Chung, C. S., \& Caldera, R. (2006). Holistic processing is finely tuned for faces of one's own race. Psychological Science, 17, 608-615.

Mondloch, C. J., Le Grand, R., \& Maurer, D. (2002). Configural face processing develops more slowly than featural face processing. Perception, 31, 553-566.
Richler, J. J., Bukach, C. M., \& GaUthier, I. (2009). Context influences holistic processing of nonface objects in the composite task. Attention, Perception, \& Psychophysics, 71, 530-540.

Richler, J. J., Gauthier, I., Wenger, M. J., \& Palmeri, T. J. (2008). Holistic processing of faces: Perceptual and decisional components. Journal of Experimental Psychology: Learning, Memory, \& Cognition, 34, 328-342.

Richler, J. J., Tanaka, J. W., Brown, D. D., \& Gauthier, I. (2008). Why does selective attention to parts fail in face processing? Journal of Experimental Psychology: Learning, Memory, \& Cognition, 34, 1356-1368.

Robbins, R., \& McKone, E. (2007). No face-like processing for objectsof-expertise in three behavioural tasks. Cognition, 10, 34-79.

Singer, M. H., Lappin, J. S., \& Moore, L. P. (1975). The interference of various word parts on color naming in the Stroop test. Perception \& Psychophysics, 18, 191-193.

TANAKA, J. W., \& FARAH, M. J. (1993). Parts and wholes in face recognition. Quarterly Journal of Experimental Psychology, 46A, 225-245.

Troje, N., \& BüLthoff, H. H. (1996). Face recognition under varying poses: The role of texture and shape. Vision Research, 36, 1761-1771.

Wenger, M. J., \& IngValson, E. M. (2002). A decisional component of holistic encoding. Journal of Experimental Psychology: Learning, Memory, \& Cognition, 28, 872-892.

Wong, A. C.-N., Palmeri, T. J., \& Gauthier, I. (in press). Conditions for face-like expertise with objects: Becoming a Ziggerin expert—but which type? Psychological Science.

Young, A., Hellawell, D., \& Hay, D. (1987). Configurational information in face perception. Perception, 16, 747-759.

(Manuscript received July 7, 2008; revision accepted for publication October 14, 2008.) 